\title{
Un modelo explicativo de las creencias y actitudes hacia las Matemáticas: Un análisis basado en modelos de ecuaciones estructurales
}

\author{
Ronny Gamboa Araya, Universidad Nacional (Costa Rica) \\ Tania Elena Moreira-Mora, Instituto Tecnológico de Costa Rica (Costa Rica)
}

Recibido el 29 de mayo de 2015; aceptado el 22 de mayo de 2016

Un modelo explicativo de las creencias y actitudes hacia las Matemáticas: Un análisis basado en modelos de ecuaciones estructurales

\section{Resumen}

Este artículo resume los resultados de una investigación cuyo propósito fue analizar qué aspectos relacionados con las creencias y actitudes de los estudiantes hacia las Matemáticas poseen una mayor evidencia de relación con ellas. Se utilizó un diseño cuantitativo. La recolección de datos se realizó con 506 estudiantes de educación secundaria de Costa Rica. Para el análisis de la información se utilizó un modelo de ecuaciones estructurales. Los resultados señalan que desde una visión de las Matemáticas de resolución de problemas, existe evidencia de relación entre los componentes cognitivo, afectivo y conductual, y la actitud hacia la disciplina. La mayoría de las tendencias didácticas del docente y la percepción de este presentaron evidencia de relación directa con las creencias hacia las Matemáticas e indirecta con las actitudes hacia la materia. Sin embargo, fue la imagen del estudiante respecto a si mismo en la disciplina la que mayor relación presentó.

Palabras clave. Creencias; actitudes; Matemáticas; estudiantes; modelo de ecuaciones estructurales.

Um modelo explicativo das crenças e atitudes em relação à matemática: Uma análise baseada em modelos de equações estruturais

\section{Resumo}

Este artigo resume os resultados de uma investigação cujo objetivo foi analisar quais os aspectos das crenças e atitudes dos alunos relativamente à matemática, ter maior evidência de relacionamento com eles. Foi utilizado o delineamento quantitativo. A coleta de dados foi realizada com 506 estudantes do ensino médio em Costa Rica. Para a análise dos dados, utilizou-se um modelo estrutural. Os resultados mostram que a partir do ponto de vista da Matemática resolver problema, há evidências de uma relação entre os componentes cognitivos, emocionais e comportamentais, e atitude para com a disciplina. A maioria dos professores de ensino tendências e percepção das provas apresentadas directamente relacionado com as crenças sobre Matemática e indireta a atitudes em relação ao assunto. No entanto, foi a imagem do aluno sobre si mesmo na disciplina que tinha uma relação mais elevada.

Palavras chave. Crenças; atitudes; Matemática; estudantes; modelo de equações estruturais.

Para citar: Gamboa, R. \& Moreira-Mora, T. E. (2016). Un modelo explicativo de las creencias y actitudes hacia las Matemáticas: Un análisis basado en modelos de ecuaciones estructurales Avances de Investigación en Educación Matemática, 10, 27 - 51 


\begin{abstract}
An explanatory model of the beliefs and attitudes towards mathematics: An analysis based on structural equation modeling
\end{abstract}

\title{
Abstract
}

This article summarizes the results of an investigation whose purpose was to analyze what aspects of the beliefs and attitudes of students towards mathematics, have greater evidence of relationship with them. A quantitative design was used. Data collection was performed with 506 high school students in Costa Rica. For the analysis of the information, a structural equation model was used. The results show that from the viewpoint of Mathematics problem solving, there is evidence of a relationship between the cognitive, emotional and behavioral components, and attitude toward discipline. Most didactic trends of mathematics' teachers and perception about him presented evidence directly related to beliefs about Mathematics and indirect to attitudes toward the subject. However, it was the image of the student about himself in the discipline which had a higher ratio.

Key words. Beliefs; attitudes; mathematics; students; structural equation model.

Un modèle explicatif des croyances et des attitudes envers les mathématiques: Une analyse basée sur la modélisation par équation structurelle

\section{Résumé}

Cet article résume les résultats d'une enquête dont le but était d'analyser les aspects des croyances et des attitudes des élèves envers les mathématiques, ont une plus grande preuve de relation avec eux. Une conception quantitative a été utilisée. La collecte des données a été réalisée avec 506 élèves du secondaire au Costa Rica. Pour l'analyse de l'information, un modèle d'équation structurelle a été utilisé. Les résultats montrent que du point de vue du problème de mathématiques problèmes, il existe des preuves d'une relation entre les composantes cognitives, émotionnelles et comportementales, et l'attitude envers la discipline. La plupart des professeurs qui enseignent les tendances et la perception de la preuve présentée directement liée à des croyances sur les mathématiques et indirecte aux attitudes envers le sujet. Cependant, il était l'image de l'étudiant sur lui-même dans la discipline qui avait un ratio plus élevé.

Paroles clés. Croyances; attitudes; Mathématiques; étudiants; modèle d'équation structurelle.

\section{Introducción}

La problemática que enfrentan la mayoría de los estudiantes en Matemáticas ha sido un tema recurrente en diferentes foros y congresos nacionales e internacionales. Muchos investigadores señalan aspectos sobre el origen de esta problemática y el mismo rechazo hacia esta disciplina que no obedecen únicamente a asuntos relacionados con su naturaleza, sino que son el resultado de una serie de estereotipos que se han creado a su alrededor y que se transmiten en el entorno familiar y educativo (Chaves, Castillo \& Gamboa, 2008).

Las distintas experiencias que un estudiante ha tenido respecto a las Matemáticas y su proceso de aprendizaje, determinan, en parte, la forma en cómo estos se desenvuelven en la disciplina. La tradicional imagen negativa que ha rodeado a esta materia ha provocado una serie de actitudes y creencias en los estudiantes que han inducido, en algunos casos, a un bloqueo cognitivo. La imagen social negativa de las Matemáticas se ve reflejada en el comportamiento de muchos estudiantes en las aulas, no sólo porque manifiestan una actitud de rechazo hacia ellas y a determinados docentes que las imparten, sino porque también hacen alarde de obtener malos 
resultados en dicha disciplina como una forma de sobresalir en el grupo de amistades (Armenteros, 2009).

Entender y plantear estrategias para atender la problemática que enfrentan los estudiantes en el aprendizaje de la disciplina implica no solo el planteamiento de tareas adecuadas sino tener en cuenta aspectos personales de los estudiantes, como sus creencias, actitudes y perfil motivacional (Berenguel, Montoro \&Moreno, 2015).

Desde este punto de vista, el factor emocional constituye un aspecto primordial en el aprendizaje como un elemento que puede explicar, al menos parcialmente, las dificultades que presentan las Matemáticas para muchas personas (Cubillo, Gamboa, Márquez, Mora, Montero \& Fuentes, 2010). En este sentido Chandía, Quiroga y Ulloa (2006) señalan que algunos estudios indican que cuando los estudiantes se enfrentan a situaciones adversas constantemente, la aparición de condiciones afectivas negativas dificulta el aprendizaje pues los discentes establecen ciertas creencias (cuyo sustento es absolutamente emocional) y las internalizan como verdades absolutas. En este sentido, autores como Blanco, Guerrero y Caballero (2013), son enfáticos al indicar la importancia de considerar en forma integrada los aspectos cognitivos y afectivos de la enseñanza de las Matemáticas y el aprendizaje en diferentes niveles, como una forma de incentivar el cambio en las creencias y actitudes de los estudiantes.

Con base en lo anterior es evidente que la interrelación existente entre la Educación Matemática y los componentes del dominio afectivo influye fuertemente en la enseñanza y aprendizaje de las Matemáticas; por ello autores como Chaves et al. (2008) señalan la importancia de tomar en cuenta los diferentes factores que configuran el dominio afectivo en los procesos educativos, en particular, las creencias $\mathrm{y}$ actitudes, $\mathrm{y}$ sus interrelaciones.

Por lo anterior, el objetivo de este trabajo es determinar cuáles de las dimensiones vinculadas con las actitudes y creencias hacia las Matemáticas presentan mayor evidencia de relación con ambos constructos, lo anterior mediante un modelo explicativo basado en un modelo de ecuaciones estructurales. Esto le permitirá a los docentes de la disciplina prestar atención a estos elementos e introducir cambios en su práctica educativa, tendientes a modificar las actitudes y creencias negativas y reforzar las positivas.

\section{Marco teórico}

Las Matemáticas se han convertido, dentro del currículo escolar, en una de las materias más temidas, lo que ha propiciado la construcción de una "imagen social negativa" que se transmite de una generación a otra. En este sentido, lo que los estudiantes piensan acerca de las Matemáticas influye en sus sentimientos hacia la materia y predispone su actuar en las distintas tareas relacionadas con la asignatura; es decir, si tienen creencias negativas acerca de las Matemáticas o su enseñanza, mostrarán sentimientos adversos hacia las tareas relacionadas con ellas tales como rechazo o indisposición (Blanco, Guerrero \& Caballero, 2013).

En la Educación Matemática, "el dominio afectivo cumple muchos significados: es un sistema regulador del conocimiento que permite al estudiante actuar, pensar y orientar su ejecución; es un indicador de las experiencias que se han adquirido a lo largo de la vida estudiantil; es una fuerza impulsora o bloqueadora hacia la actividad matemática y un medio para transmitir el conocimiento matemático" (Domínguez \& Jarero, 2010, p. 191). 
Al respecto, Álvarez (2007) indica que

la experiencia de los docentes confirma la importancia de la afectividad en el aprendizaje, al observar como los trastornos emocionales limitan el proceso de enseñanza aprendizaje; estudiantes ansiosos, angustiados, deprimidos, pesimistas, frustrados no logran aprender de manera adecuada, en cambio, aquellos alumnos sosegados, optimistas, alegres, tranquilos y con elevada confianza en sí mismos logran aprender con más facilidad. (p. 91).

En este sentido, la motivación, la valoración o la disposición hacia las Matemáticas han sido uno de los factores endógenos asociados al rendimiento escolar, al igual que las actitudes y aptitudes del estudiantado (Moreira, 2009). Relacionado con lo anterior, Demicheli (2009) indica que las creencias y las actitudes son constructos teóricos fundamentales en el estudio, descripción, explicación y predicción de la conducta de los individuos, pues representan un indicio de sus experiencias pasadas y poseen influencia directa sobre su quehacer diario y proyección hacia el futuro.

Gran parte de las dificultades presentes en la enseñanza y aprendizaje de las Matemáticas tienen mucho que ver con la representación social que los estudiantes tienen de las mismas (Naranjo \& Segura, 2010). Con una adecuada comprensión de esta situación se pueden plantear estrategias de enseñanza acordes a dicho "contexto emocional", que permitiría estimular las valoraciones positivas hacia la materia y modificar aquellos aspectos del quehacer de aula que provocan reacciones negativas hacia las Matemáticas. Esto le posibilitaría al docente realizar una autoreflexión sobre su papel en la formación de determinadas valoraciones de sus estudiantes, y en las propias, respecto a la asignatura.

Las actitudes pueden considerarse como representaciones psicológicas de la influencia que ejerce cada sociedad y cultura sobre las personas en tanto individuos. Por un lado, son inseparables de los contextos que las generan, mantienen y actualizan bajo ciertas condiciones, pero al mismo tiempo son una expresión que articula un conjunto más o menos amplio de experiencias individuales únicas (Demicheli, 2009, pp. 95-96).

Existen tres componentes que constituyen una actitud: el cognitivo, que incluye las ideas y percepciones sobre el objeto de la actitud; el afectivo, que corresponde a los sentimientos que el sujeto tiene y la intensidad de los mismos; y el conductual, dada por la respuesta que el sujeto tiene en reacción al objeto de la actitud (Abraham, Mena, Rodríguez, Golbach, Rodríguez \& Galindo, 2010). En el caso de las Matemáticas, Cárdenas (2008) apunta que las actitudes se pueden clasificar en dos categorías

a) Actitudes hacia las Matemáticas, relacionadas con un conjunto de disposiciones que manifiesta el individuo para aceptar o no, familiarizarse o no, con determinados contenidos de la disciplina (Petriz, Barona, López \& Quiroz, 2010). Pueden referirse a cualquiera de los siguientes aspectos:

i) Actitud hacia las Matemáticas y los matemáticos (aspectos sociales).

ii) Interés por el trabajo matemático, científico.

iii) Actitud hacia las Matemáticas como asignatura.

iv) Actitud hacia determinadas partes de las Matemáticas.

v) Actitud hacia los métodos de enseñanza.

b) Actitudes matemáticas, las cuales poseen carácter cognitivo y se refieren al modo de utilizar capacidades como la flexibilidad de pensamiento, la apertura mental, el espíritu crítico, la objetividad, entre otras, consideradas como importantes para el aprendizaje de la disciplina. 
El estudio de las actitudes hacia las Matemáticas es importante debido a que el desarrollo de actitudes positivas debe ser un objetivo de los sistemas educativos como un requisito para mejorar el aprendizaje de los estudiantes, muestran el ambiente de la escuela y del contexto social en el cual se da la enseñanza y el aprendizaje de la disciplina y porque la literatura ha sugerido que hay una relación positiva entre las actitudes hacia las Matemáticas y la capacidad académica (Cárdenas, 2008).

Respecto al concepto de creencia se señala que esta

es el conjunto de puntos de vista, de representaciones subjetivas, que la persona va interiorizando (individualizando) y reforzando o debilitando en el decursar de su vida. Este sistema establece el contexto dentro del cual los recursos, la heurística y el control funcionan" (Sánchez, 2008, p. 3).

En Educación Matemática, Parra (2005) señala que una creencia se entiende como el conjunto de conocimientos, valores e ideologías que posee un estudiante acerca de la disciplina. En los estudios de McLeod (1989, 1992), citados por McLeod y McLeod (2002), se logró determinar que las creencias individuales de los estudiantes sobre las Matemáticas y su enseñanza, sobre sí mismo en su rol de aprendiz o sobre el contexto social, tienen un fuerte impacto en el proceso educativo. Este autor menciona dos categorías: creencias sobre las Matemáticas como disciplina y creencias sobre sí mismo y su relación con las Matemáticas

Por su parte, Sánchez (2008) indica que las creencias de los estudiantes en las Matemáticas están relacionadas con cuatro ejes: sobre sí mismo; sobre el entorno (papel que cree jugar o desempeñar ante su profesor, compañeros, entre otros); sobre el problema (si es capaz de resolverlo y las reglas para hacerlo); sobre las Matemáticas. Otros autores como Op't Eynde, De Corte y Verschaffel (2002) indican que las creencias de los estudiantes sobre la Educación Matemática están situadas en un determinado contexto, en el cual ellos participan, y están influenciadas por sus necesidades psicológicas, deseos, metas, entre otras. De esta manera, los sistemas de creencias de los estudiantes relacionados con las Matemáticas están constituidos por sus creencias sobre el contexto de la clase, sobre sí mismos y sobre la Educación Matemática.

En este sentido Op't Eynde et al. (2002) desarrollaron una estructura de las creencias de los estudiantes. Para ello establecen las siguientes subcategorías:

\section{a) Creencias sobre la Educación Matemática}

Estas incluyen las creencias de los estudiantes sobre las Matemáticas, el aprendizaje, la resolución de problemas matemáticos y la enseñanza de las Matemáticas. Esta categoría, según Estrada (2002), considera la naturaleza de las Matemáticas donde los aspectos eminentemente afectivos son menos importantes.

Al respecto, Ernest (1988) señala que, en cuanto a la naturaleza de las Matemáticas, existen tres visiones:

i) Instrumentalista: la cual considera que las Matemáticas constituyen una acumulación de hechos, reglas y habilidades que pueden ser usadas en la ejecución de algún fin externo.

ii) Platónica: concibe que las Matemáticas son un cuerpo de conocimientos estático y unificado; son descubiertas, no creadas.

iii) Resolución de problemas: visualiza las Matemáticas como un campo de creación e invención humana en continua expansión, que son un producto cultural no acabado y sus resultados están abiertos a la revisión. 


\section{b) Creencias de los estudiantes sobre sí mismos}

En esta categoría se incluyen las creencias sobre la autoeficacia, el control, el valor de la tarea y la orientación de las metas relacionadas con las Matemáticas. Incorpora elementos relacionados con la confianza y el autoconcepto, el cual se convierte en un buen predictor del rendimiento académico en la disciplina (Gil, Blanco \& Guerrero, 2005).

\section{c) Creencias de los estudiantes sobre su contexto}

Esta categoría de las creencias hacia las Matemáticas agrupa aquellas relacionadas con las normas y las prácticas sociomatemáticas en la clase, el papel y funcionamiento de su profesor y el rol y desempeño de los estudiantes.

Sobre este aspecto, Contreras $(1998,2009)$ plantea cuatro tendencias didácticas que rigen la práctica docente: tradicionalista, tecnológica, espontaneísta e investigativa. De forma más específica, Contreras (1998) caracteriza cada una de estas tendencias según la metodología, sentido de la asignatura, concepción del aprendizaje, papel del alumno, papel del profesor y evaluación.

i) Tradicionalista, que se caracteriza por el uso de la exposición magistral y el libro de texto como material curricular. Bajo esta tendencia el profesor se basa en una programación prescrita con antelación, externa a él y rígida. Las lecciones se basan en la adquisición de conceptos, utilizando la memoria como único recurso. Se parte del hecho de que el alumno adquiere los conocimientos a partir de la exposición que el profesor realiza, por lo que él es el único responsable de los resultados del aprendizaje. El docente cree que la evaluación es una actividad que se realiza al final del proceso educativo con el objetivo de medir su capacidad de retener información, donde el examen es el instrumento ideal para medir dicho aprendizaje.

ii) Tecnológica, en la cual el profesor no expone los contenidos en su fase final, sino que simula su proceso de construcción, apoyándose en estrategias expositivas. Bajo esta tendencia interesan tanto los conceptos como los procesos lógicos que los sustentan. Parte del principio que el aprendizaje se realiza utilizando la memoria, cuya organización se da según la lógica de la disciplina; así, para que el estudiante aprenda debe entender y asimilar el conocimiento que proviene del exterior. Se considera al alumno como el principal responsable de los resultados del aprendizaje, siempre que el contexto elegido por el profesor sea adecuado. En esta tendencia el docente cuestiona el proceso de aprendizaje a la luz de los resultados obtenidos. El examen es el instrumento ideal para medir dicho aprendizaje pero no necesariamente deben coincidir con el final del desarrollo de todos los contenidos.

iii) Espontaneista, la cual se caracteriza por que el docente propone actividades de manipulación de modelos, a través de las cuales se espera que se produzca un conocimiento no organizado. La programación se basa en los intereses que poseen los alumnos y en la negociación con ellos, por lo que no implica una organización inicial. Interesan tanto los conceptos como los procedimientos y el fomento de actitudes positivas hacia el trabajo escolar. La asignatura posee un carácter formativo, con objeto de servir de instrumento para un cambio actitudinal del alumno, por lo que la adquisición de valores racionales le permiten conformar una actitud lógica ante los problemas cotidianos. El profesor considera que el estudiante aprende cuando el objeto de aprendizaje, que surge aleatoriamente del contexto, posee un significado para el alumno, por ello este induce al alumno a participar en las actividades que promueve; además, concibe la evaluación como una forma permanente de "medir" el aprendizaje 
que le aporta elementos para reorientarlo si es requerido. El examen tiene connotaciones de índole psicológica que influyen desfavorablemente en la actividad del alumno y en las relaciones personales dentro del aula.

iv) Investigativa, en la cual el profesor organiza el proceso que guiará al estudiante a la adquisición de los conocimientos determinados a través de su investigación. Así, interesa tanto la adquisición de conceptos como el desarrollo de procedimientos y el fomento de actitudes positivas hacia la propia materia y el trabajo escolar, donde el objetivo de la asignatura es dotar al alumno de herramientas que le permitan el aprendizaje autónomo. El profesor piensa que el aprendizaje se produce a través de investigaciones que han sido planificadas por él, donde es necesario que el alumno otorgue significado a lo que aprende y sea consciente de su propio proceso de aprendizaje. En esta tendencia el profesor concibe la evaluación como una manera permanente del "medir" el aprendizaje, lo que le aporta información para reconducirlo en cada momento y orientar la enseñanza hacia los aprendizajes previstos a través de contextos más apropiados; el examen es un instrumento que posee la finalidad de aprendizaje (actividad individual) y de control de dicho proceso.

En la siguiente sección se aborda algunos aspectos metodológicos de la investigación.

\section{Método}

\subsection{Población}

En la investigación participaron todos los estudiantes de décimo año de tres instituciones académicas públicas diurnas de la región educativa de la provincia de Heredia, Costa Rica. Estas instituciones fueron seleccionadas por la similitud de sus características relacionadas con su ubicación geográfica, población estudiantil, cantidad de docentes, entre otros. En cada una de las instituciones se trabajó con todas las secciones de décimo año de estos colegios, por lo que no se utilizó ninguna muestra. En total fueron 506 estudiantes de los cuales 231 (45.7\%) son hombres y 274 $(54.2 \%)$ mujeres. La edad de los discentes osciló entre 15 y 20 años. La mayoría de ellos tenían 16 años (53.6\% de la población).

\subsection{Instrumento}

Para la recolección de los datos se utilizó un cuestionario. Para su confección se tomaron como base los supuestos teóricos relacionados con las creencias y actitudes hacia las Matemáticas y los instrumentos aplicados en otras investigaciones. El instrumento consistió en una escala tipo Likert con cinco valores escalares. Estaba compuesto de 127 preguntas, 5 de ellas correspondían a información general, 47 con actitudes hacia las Matemáticas y 75 relacionadas con las creencias hacia la disciplina.

Respecto a las actitudes hacia las Matemáticas, los ítems medían tres componentes: cognitivo, afectivo y conductual. En el caso de las creencias hacia la disciplina se plantearon preguntas referidas a las siguientes dimensiones:

a) Creencias sobre las Matemáticas según tres visiones:

- instrumentalista,

- platónica,

- resolución de problemas. 
b) Creencias de los individuos respecto a sí mismos en Matemáticas.

c) Creencias sobre el papel del profesor dentro del contexto de la clase, con base en

- la tendencia didáctica del docente, clasificada en: tradicionalista, tecnológica, espontaneísta, investigativa.

- Imagen de un buen profesor de Matemáticas.

- Percepción del docente.

Para obtener evidencias de validez de contenido de los instrumentos se recurrió a la consulta constante de la teoría relacionada con el tema de investigación, en conjunción con el criterio de jueces. El promedio de concordancia de los jueces por ítem fue de $84.5 \%$, según el índice de Kappa de Fleiss.

Posterior al criterio de jueces se llevó a cabo una prueba piloto con la participación de 55 estudiantes de décimo de la provincia de Heredia. Ninguno de los participantes pertenecía a la población en la cual se desarrolló la investigación. Una vez aplicados los cuestionarios se procedió a la digitación de los datos y se realizó el análisis del Alfa de Cronbach para cada uno de los componentes de las actitudes hacia las Matemáticas y cada una de las dimensiones en las que se clasificó las creencias hacia la disciplina, cuyos índices fueron:

a) Componente cognitivo: 0,900

b) Componente afectivo: 0,901

c) Componente conductual: 0,883

d) Creencia sobre las Matemáticas: 0,724

e) Creencias sobre los individuos respecto a sí mismos en Matemáticas: 0,845

f) Creencias sobre el papel del profesor dentro del contexto de la clase: 0,923

El Alfa de Cronbach para todos los ítems relacionados con las actitudes hacia las Matemáticas fue de 0,960 y para las creencias hacia la disciplina fue de 0,921.

Una vez efectuado el análisis anterior se realizó un estudio por componentes principales, con el propósito de evaluar si cada uno de los factores o categorías establecidas estaban representados por los ítems propuestos y verificar que cada ítem estuviera asociado a un solo factor o categoría. Lo anterior permitió depurar las escalas al identificar los ítems multidimensionales y con baja discriminación.

\subsection{Hipótesis y construcción del modelo de ecuaciones estructurales}

El propósito de la investigación con el planteamiento de un modelo de ecuaciones estructurales fue analizar la relación de causalidad entre las variables que inciden en las creencias de los estudiantes hacia las Matemáticas, tales como las creencias sobre la disciplina, de los individuos respecto a sí mismos en la materia y sobre el papel del profesor dentro del contexto de la clase. A la vez, determinar la relación entre las creencias con las actitudes, las cuales también están mediadas por el componente cognitivo, afectivo y conductual.

Por lo tanto, las hipótesis que se pretendieron comprobar para los estudiantes participantes en el estudio, fueron

1. Hay relación entre los componentes cognitivo, afectivo, conductual y la actitud hacia las Matemáticas. 
2. Hay relación entre la visión de las Matemáticas y las creencias hacia la disciplina.

3. Hay relación entre la imagen de sí mismo en Matemáticas y las creencias hacia la disciplina.

4. Hay relación entre la tendencia didáctica del profesor de Matemáticas y las creencias hacia la disciplina.

5. Hay relación entre la imagen de un buen profesor de Matemáticas y las creencias hacia la disciplina.

6. Hay relación entre la percepción de los estudiantes sobre el profesor de Matemáticas y las creencias hacia la disciplina.

7. Hay relación entre las creencias y actitudes hacia las Matemáticas.

El modelo de ecuaciones estructurales se analizó por medio del software Lisrel (linear structural relations), versión 9.1. La tabla 1 muestra el nombre dado a cada una de las dimensiones consideradas en el modelo de ecuaciones estructurales.

Tabla 1. Nombre dado a las dimensiones relacionadas con las actitudes y creencias de los estudiantes hacia las Matemáticas.

\begin{tabular}{|c|c|c|c|}
\hline \multicolumn{3}{|c|}{ Dimensión } & Nombre \\
\hline \multicolumn{3}{|l|}{ Cognitivo } & C.Cognit \\
\hline \multicolumn{3}{|l|}{ Afectivo } & C.Afecti \\
\hline \multicolumn{3}{|l|}{ Conductual } & C.Conduc \\
\hline \multirow{2}{*}{\multicolumn{3}{|c|}{ Actitudes hacia las Matemáticas }} & Actitud \\
\hline & & & V.Instru \\
\hline \multicolumn{3}{|c|}{ Creencia sobre las Matemáticas } & V.Platon \\
\hline \multirow{2}{*}{\multicolumn{3}{|c|}{$\begin{array}{c}\text { Resolución de problemas } \\
\text { Creencias de los individuos respecto a sí mismos en Matemáticas }\end{array}$}} & V.Resolu \\
\hline & & & Si_mismo \\
\hline \multirow{5}{*}{\multicolumn{3}{|c|}{$\begin{array}{lll}\text { Creencias asociadas al } & \begin{array}{l}\text { Sobre su tendencia } \\
\text { didáctica }\end{array} & \begin{array}{l}\text { Tradicionalista } \\
\text { Tecnológica } \\
\text { papel del profesor en la } \\
\text { clase }\end{array} \\
& \begin{array}{l}\text { Espontaneísta } \\
\text { Investigativa }\end{array} \\
\text { Percepción del docente }\end{array}$}} & C.Tradic \\
\hline & & & $\begin{array}{l}\text { C.Tecnol } \\
\text { C.Espont } \\
\text { C.Invest }\end{array}$ \\
\hline & & & I.B.Prof \\
\hline & & & P.docent \\
\hline & & & Creencia \\
\hline
\end{tabular}

Para la construcción del modelo se utilizaron los siguientes supuestos y relaciones. Como se mencionó, las actitudes hacia las Matemáticas se constituyen a partir de tres componentes: cognitivo, afectivo y conductual. Por esta razón se establecieron las siguientes relaciones (Figura 1):

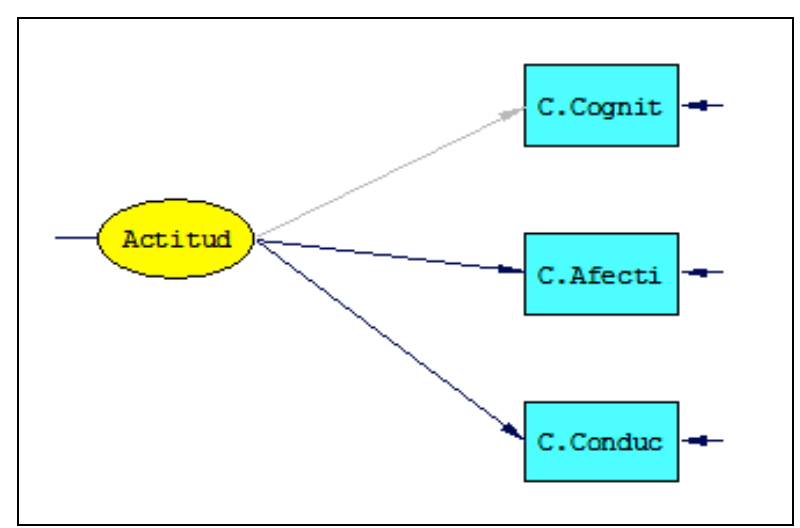


Figura 1. Representación de los indicadores asociados al constructo Actitud (modelo de medida para $y$ ).

En el caso de las creencias hacia la disciplina estas se consideraron desde varios componentes: creencias sobre las Matemáticas, clasificadas en tres visiones (instrumentalista, platónica y de resolución de problemas), creencias de los individuos respecto a sí mismos en Matemáticas y creencias asociadas al papel del profesor dentro del contexto de la clase, según su tendencia didáctica (clasificada en tradicionalista, tecnológica, espontaneísta e investigativa), imagen de un buen profesor de Matemáticas y percepción del docente.

Debido a que las visiones de las Matemáticas y las concepciones didácticas del docente se clasificaron en tres y cuatro divisiones, respectivamente, los modelos propuestos consideraron las posibles combinaciones de ellas. Por ejemplo, para la visión instrumentalista se tendrían las siguientes combinaciones: instrumentalistatradicionalista, instrumentalista-tecnológica, instrumentalista-espontaneísta, instrumentalista-investigativa. Similarmente para las otras visiones de las Matemáticas.

Las relaciones que se establecieron fueron (Figura 2)

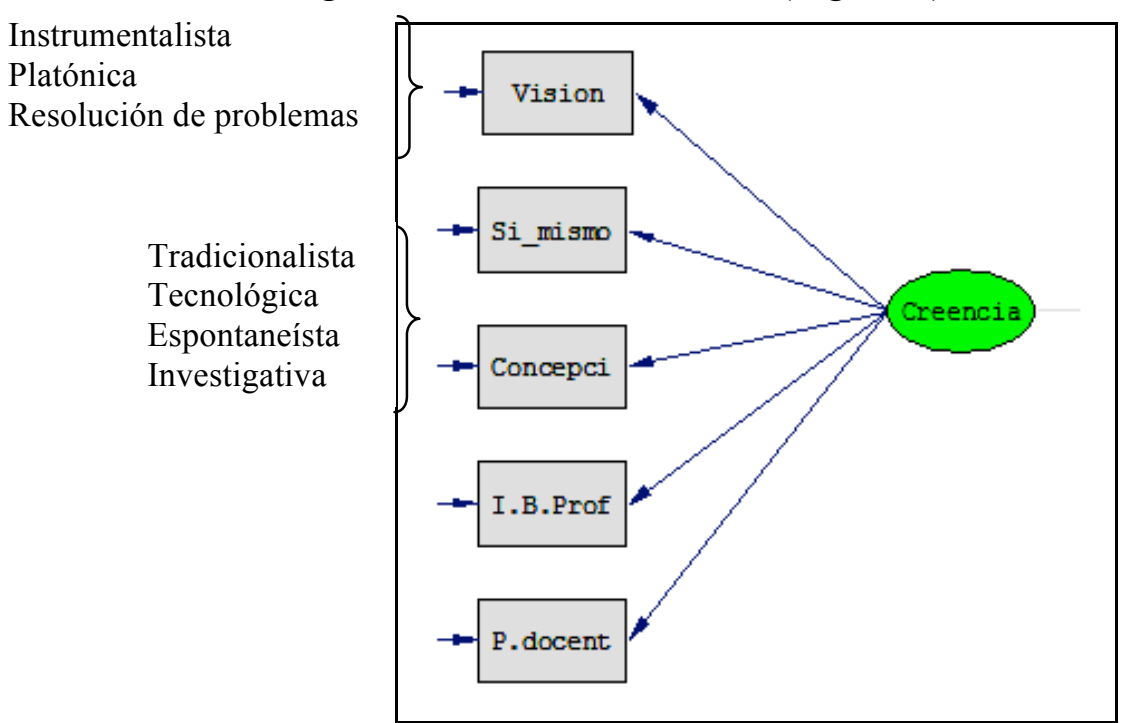

Figura 2. Representación de los indicadores asociados al constructo Creencia (modelo de medida para $x$ ).

Luego, Mora y Barrantes (2008) indican que las concepciones acerca de la disciplina poseen influencia en las creencias de los diferentes aspectos que tienen que ver con las Matemáticas y que las visiones señalas por Ernest (1988) (instrumentalista, platónica y resolución de problemas) estimulan estructuras e ideas que inciden en el proceso de enseñanza y aprendizaje de esta disciplina. Por ello, se establece en el modelo propuesto una relación entre la visión de las Matemáticas y la imagen de un buen docente de la disciplina. Esto se ejemplifica como sigue (Figura 3): 


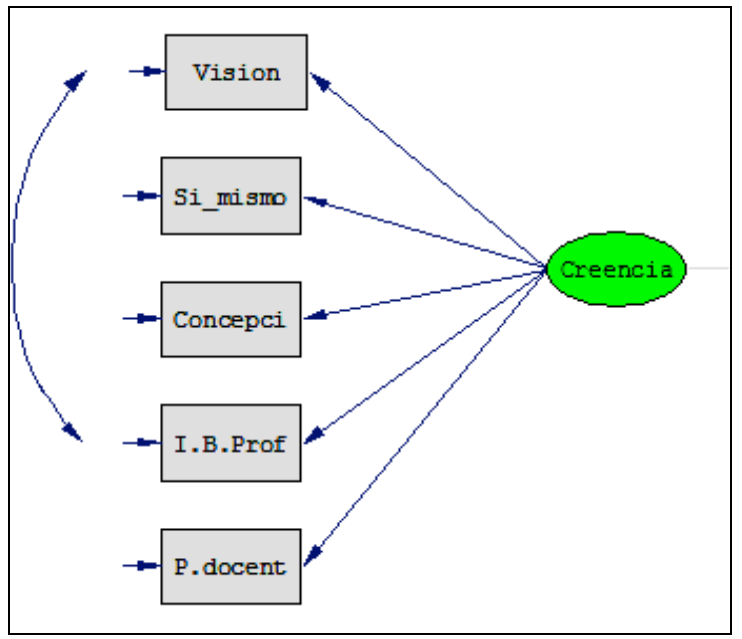

Figura 3. Representación de la relación entre los indicadores visión de las Matemáticas (Vision) e imagen de un buen profesor de Matemáticas (I.B.Prof) asociados al constructo Creencia (modelo de medida para $x$ ).

Por otra parte, las tendencias didácticas planteadas por Contreras $(1998,2009)$ y la caracterización que se realiza de ellas implican que aquella en la que el estudiante clasifique al docente se relaciona con la percepción que este posea del profesor, actividades que realiza, instrumentos de evaluación que emplea, entre otros. Por lo tanto, se puede establecer la siguiente relación (Figura 4):

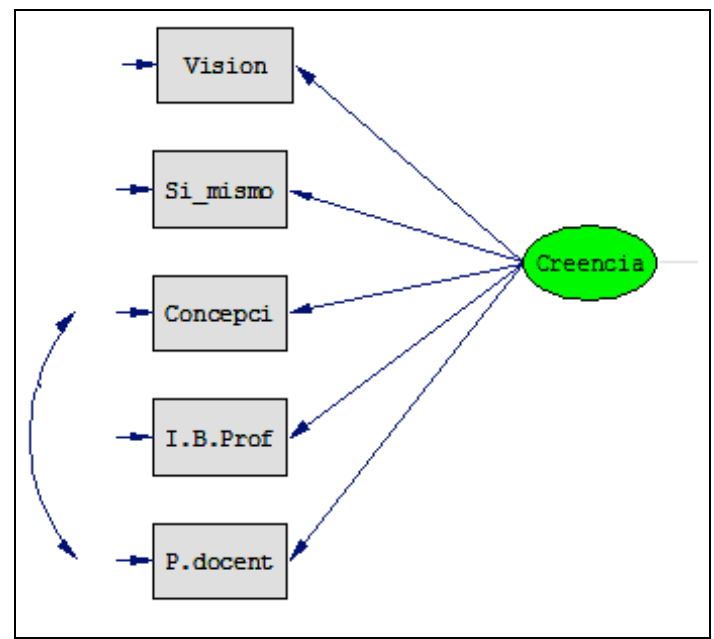

Figura 4. Representación de la relación entre los indicadores tendencia didáctica del profesor de Matemáticas (Concepci) y percepción docente (P.docent) asociados al constructo Creencia (modelo de medida para $x$ ).

Por último, según lo expuesto por Estrada (2002), Chaves et al. (2008) y Sánchez, Becerra, García y Contreras (2010) las creencias de los estudiantes hacia las Matemáticas están relacionadas con las actitudes hacia la disciplina. Incluso Gómez (2000) establece que es una relación cíclica. Sin embargo, para el modelo propuesto se considerará únicamente el efecto que las primeras poseen en las segundas (Figura 5), es decir, se considerará que "las creencias que sostiene el sujeto tiene consecuencia directa en su comportamiento en situaciones de aprendizaje y en su capacidad para aprender" (Gómez, 2000, p. 26). 


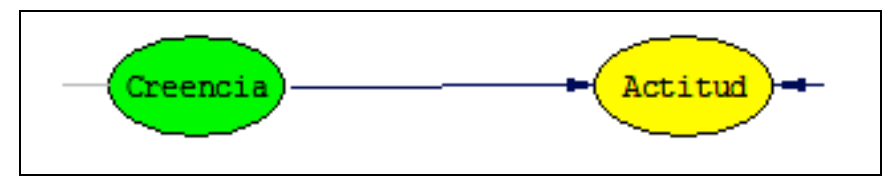

Figura 5. Representación de la relación entre los constructos Creencia y Actitud (modelo estructural).

De esta forma el modelo propuesto en esta investigación se sintetiza en la figura 6:

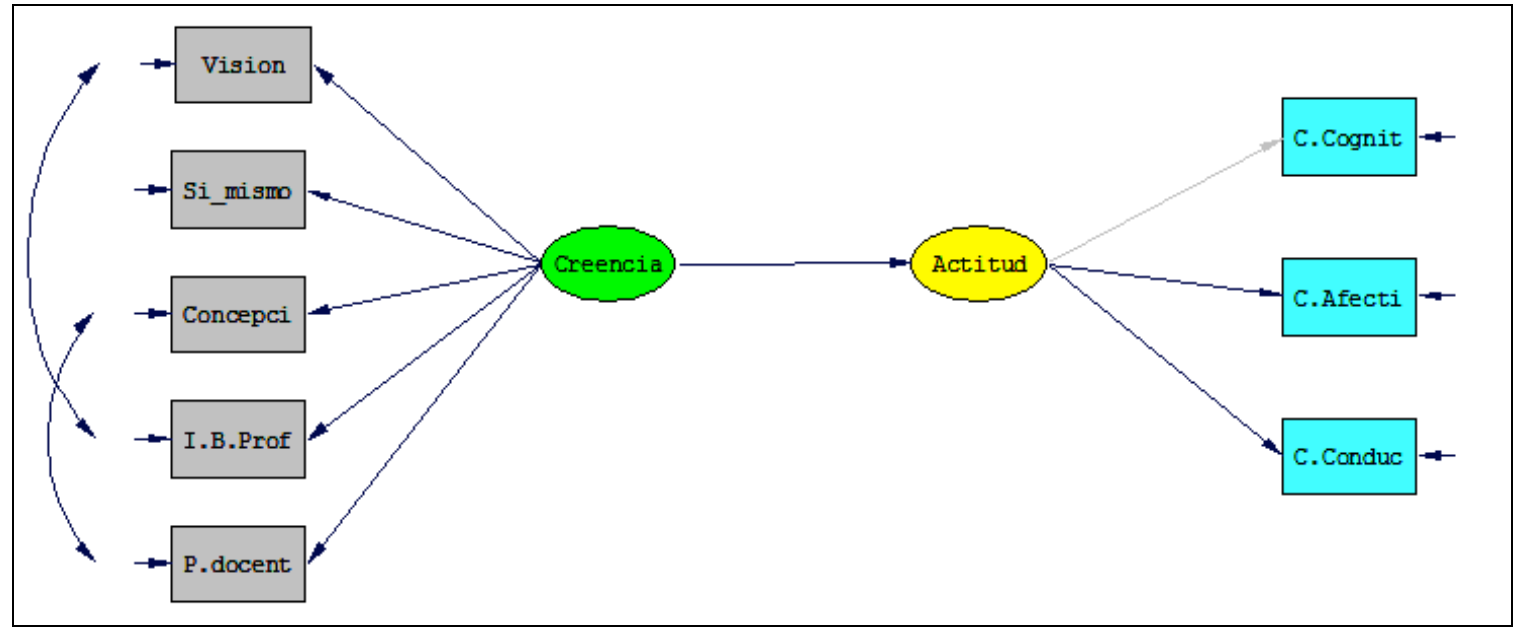

Figura 6. Modelo de ecuaciones estructurales propuesto.

Por lo tanto, de acuerdo con Diamantopoulo y Siguaw (2000), Casas (2002), Catena, Ramos y Trujillo (2003), Cea (2004) y Zamora (2012), el modelo de ecuaciones estructurales está compuesto por dos sub-modelos que pueden expresarse de forma matricial de la siguiente forma

a) Variable latente exógena: Creencia.

b) Variable latente endógena: Actitud.

c) Variables observadas exógenas: Vision (V.Instru, V.Platon, V.Resolu), Si_mismo, Concepci (C.Tradic, C.Tecnol, C.Espont, C.Invest), I.B.Prof y P.docent.

d) Variables observadas endógenas: C.Cognit, C.Afecti, C.Conduc.

e) El modelo estructural sería $\eta_{1}=\gamma_{11} \xi_{1}+\varsigma_{1}$ con

$\eta_{1}$ : representa el constructo de actitud de los estudiantes hacia las Matemáticas (Actitud).

$\xi_{1}$ : representa el constructo de creencias hacia las Matemáticas (Creencia).

$\gamma_{11}$ : representa el coeficiente que relaciona la variable endógena Actitud con la variable exógena Creencia.

$\varsigma_{1}$ : representa los errores en la ecuación definida por la variable endógena Actitud.

f) El modelo de medida para $x$ es $x_{i}=\lambda_{i 1} \xi_{1}+\delta_{i}$, donde

$x_{i}$ : representa la variable observada exógena relacionada con las creencias hacia las Matemáticas (Creencia).

$\xi_{1}$ : representa el constructo de creencias hacia las Matemáticas (Creencia).

$\lambda_{i 1}$ : representa el coeficiente que relaciona a la variable exógena Creencia con la variable observada exógena $x_{i}$. 
$\delta_{i}$ : representa las medidas de error para la variable observada exógena $x_{i}$.

g) El modelo de medida para $y$ es $y_{j}=\lambda_{j 1} \eta_{1}+\varepsilon_{j}$, donde

$y_{j}$ : representa la variable observada endógena relacionada con la actitud de los estudiantes hacia las Matemáticas (Actitud).

$\eta_{1}$ : representa el constructo de actitud de los estudiantes hacia las Matemáticas (Actitud).

$\lambda_{j 1}$ : representa el coeficiente que relaciona a la variable endógena Actitud con la variable observada endógena $y_{j}$.

$\varepsilon_{j}$ : representa las medidas de error para la variable observada endógena $y_{j}$.

Es importante señalar que según lo expresado por Catena et al. (2003) el modelo propuesto está identificado, pues posee 5 variables observadas exógenas, 3 variables observadas endógenas, lo que equivale a 36 ecuaciones; que al restarle los 19 parámetros a estimar, da como resultado 17, que coincide con los grados de libertad del modelo.

\section{Resultados}

Uno de los principales supuestos sobre los que se basa el modelo de ecuaciones estructurales es que las variables observadas, de forma conjunta, posean una distribución normal multivariante (González, Abad \& Lèvy, 2006). En este sentido, los autores indican que el hecho de cada una de estas variables verifique la normalidad univariante es una condición necesaria, pero no suficiente, para que conjuntamente tengan una distribución normal multivariante. Por lo tanto, es necesario primero comprobar que las variables, individualmente, se distribuyen normalmente y luego verificar que todas ellas en conjunto cumplen la normalidad multivariante. Entre los procedimientos que González et al. (2006) mencionan para valorar la normalidad de los datos se citan los contrastes de asimetría y curtosis.

Según lo expresado por González et al. (2006), al realizar el estudio de la normalidad univariante y multivariante en el programa Lisrel, con un nivel de error del $5 \%$, un valor experimental de $z$, para la simetría y curtosis, superior en valor absoluto a 1,96 permite rechazar la hipótesis nula que la distribución es simétrica y que posee un apuntamiento igual que la normal. Similarmente, un valor de chi cuadrado superior a 5,99 permite rechazar la hipótesis de que la simetría y curtosis es igual que la de una distribución normal, con un nivel de error del 5\%.

Al realizar el análisis de la normalidad univariante, con Lisrel, para las variables contempladas en el modelo de ecuaciones estructurales, se obtuvieron los datos que se presentan en la figura 7. 


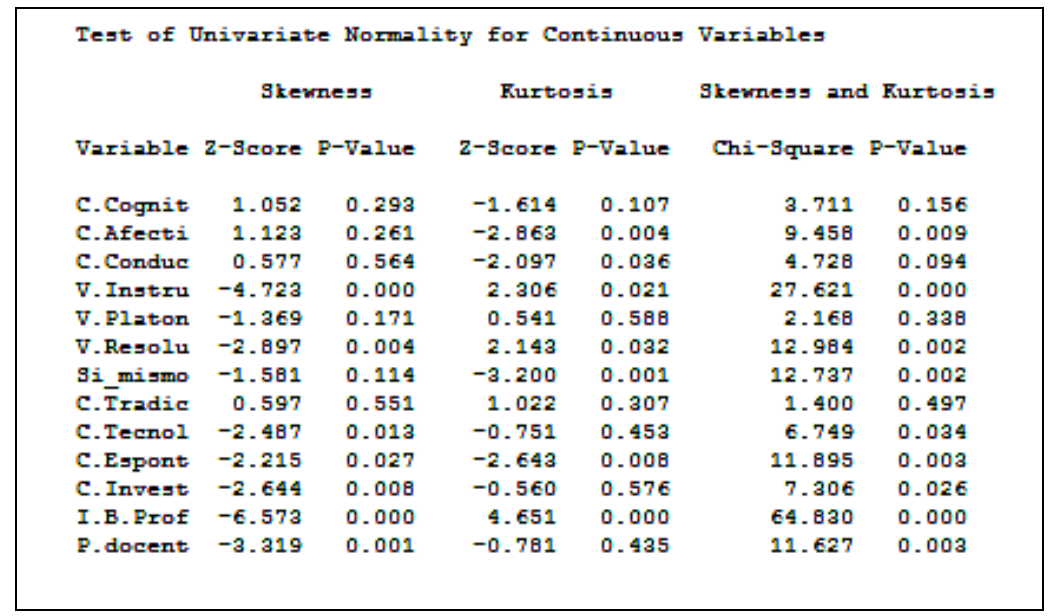

Figura 7. Análisis de la normalidad univariante con el programa Lisrel para las variables contenidas en el modelo de ecuaciones estructurales.

Como se puede observar, para un nivel de error del 5\%, la hipótesis de simetría se rechaza para las variables V.Instru, V.Resolu, C.Tecnol, C.Espont, C.Invest, I.B.Prof y P.docent; por su parte, la hipótesis de curtosis igual a la distribución normal se rechaza para C.Afecti, C.Conduc, V.Instru, V.Resolu, Si_mismo, C.Espont, I.B.Prof. Según el contraste conjunto de asimetría y curtosis, se rechaza la normalidad de las variables C.Afecti, C.Conduc, V.Instru, V.Resolu, Si_mismo, C.Tecnol, C.Espont, C.Invest, I.B.Prof y P.docent. En la figura 8 se presentan los resultados del análisis de normalidad multivariante, donde se puede observar que los contrastes de simetría y curtosis multivariante, tanto en forma individual como en conjunto, rechazan la hipótesis nula de distribución normal multivariante.

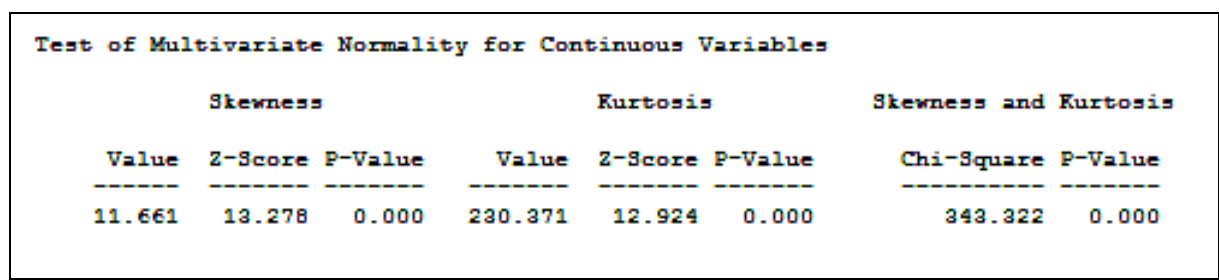

Figura 8. Análisis de la normalidad multivariante con el programa Lisrel para las variables contenidas en el modelo de ecuaciones estructurales.

La normalidad multivariante es un supuesto importante dado que al ser los tests de ajuste, en general, variaciones de chi-cuadrado, pequeñas faltas de normalidad pueden traducirse en grandes cambios en este. Sin embargo, Catena et al. (2003) y Cea (2004) apuntan que existen índices alternativos cuando se sospecha no normalidad, entre el que se destaca el índice de Bentler-Satorra, que se basa en chi-cuadrado, pero trata de corregir el sesgo que se produce por la no normalidad de los datos. Por tal razón, y ante los resultados de normalidad multivariante obtenidos, el índice de Bentler-Satorra fue utilizado en el modelo de ecuaciones estructurales.

\subsection{Evaluación del modelo}

Debido a que en este caso se trabajó con 506 estudiantes y a que no se cumplía el supuesto de normalidad multivariante (por lo que se usó el índice de Bentler-Satorra), 
el valor de la prueba Chi-cuadrado $\chi^{2}$ y el cociente $\chi^{2} / g l$ no se podrían considerar como un buen referente para determinar un buen ajuste. Por lo tanto, siguiendo la recomendación de Orgaz (2008) y Fernández (2008) se requirió complementar con otros índices de ajuste.

Para la evaluación del modelo se utilizaron la prueba Chi-cuadrado $\left(\chi^{2}\right)$, el cociente entre el valor de $\chi^{2}$ y los grados de libertad $\left(\chi^{2} / g l\right)$, los índices de bondad de ajuste (GFI), bondad de ajuste ajustado (AGFI), ajuste normado (NFI), ajuste no normativo (NNFI), ajuste comparativo (CFI), el error de la raíz cuadrada media de aproximación (RMSEA) y la raíz cuadrada media residual estandarizada (SRMR). (Cea, 2004; Orgaz, 2008; Catena et al., 2003; Zamora, 2012; Diamantopoulo \& Siguaw, 2000; Ruiz, Pardo \& San Martín, 2010; Padrós-Blázquez, Herrera-Guzmán \& Gudayol-Ferré, 2012; Calabuig, Crespo \& Mundina; 2012).

Los valores anteriores se obtuvieron para los doce modelos según las duplas establecidas. Para el caso de las siguientes duplas V.Instru-C.Tradic y V.InstruC.Tecnol, el valor del los índices de $\chi^{2} / \mathrm{gl}$, AGFI y RMSEA no cumplieron con el criterio de aceptación para un buen ajuste. En el caso de las parejas V.Instru-C.Espont y V.Instru-C.Invest, son los índices $\chi^{2} / g l$ y RMSEA los que no estuvieron acorde con la regla de admisión definida.

Para las parejas V.Platon-C.Tradic, V.Platon-C.Tecnol, V.Platon-C.Espont y V.Platon-C.Invest, los índices cumplieron con los criterios de aceptación pero los resultados obtenidos en el modelo de ecuaciones estructurales no apoyan la teoría en la cual se basan las relaciones descritas. Por ejemplo, en los cuatro casos se interpreta que las creencias y actitudes de los estudiantes hacia las Matemáticas poseen una relación inversa, es decir, que entre creencias más positivas hacia la disciplina peor es la actitud. Este hecho permitió establecer que las evidencias de estos modelos no apoyan o no se ajustan al modelo teórico.

Tres posibles razones por las cuales los modelos anteriores, relacionados con la visión platónica, no presentaron un buen ajuste se destacan a continuación. Primero, en la investigación no se consideraron la influencia de otras variables como la familia, los compañeros, entre otras, que permitieran establecer otro tipo de relaciones. Segundo, en mayor porcentaje los estudiantes se identificaron con la visión de resolución de problemas y no con la platónica. Tercero, el modelo propuesto es uno de los posibles y pueden existir otros que presenten un mejor ajuste o expliquen otras relaciones no consideradas.

Por el contrario, la visión de las Matemáticas desde una concepción de resolución de problemas presentó un ajuste adecuado para las cuatro tendencias didácticas del docente, las cuales se presentan en las figuras 9, 10, 11 y 12 . 


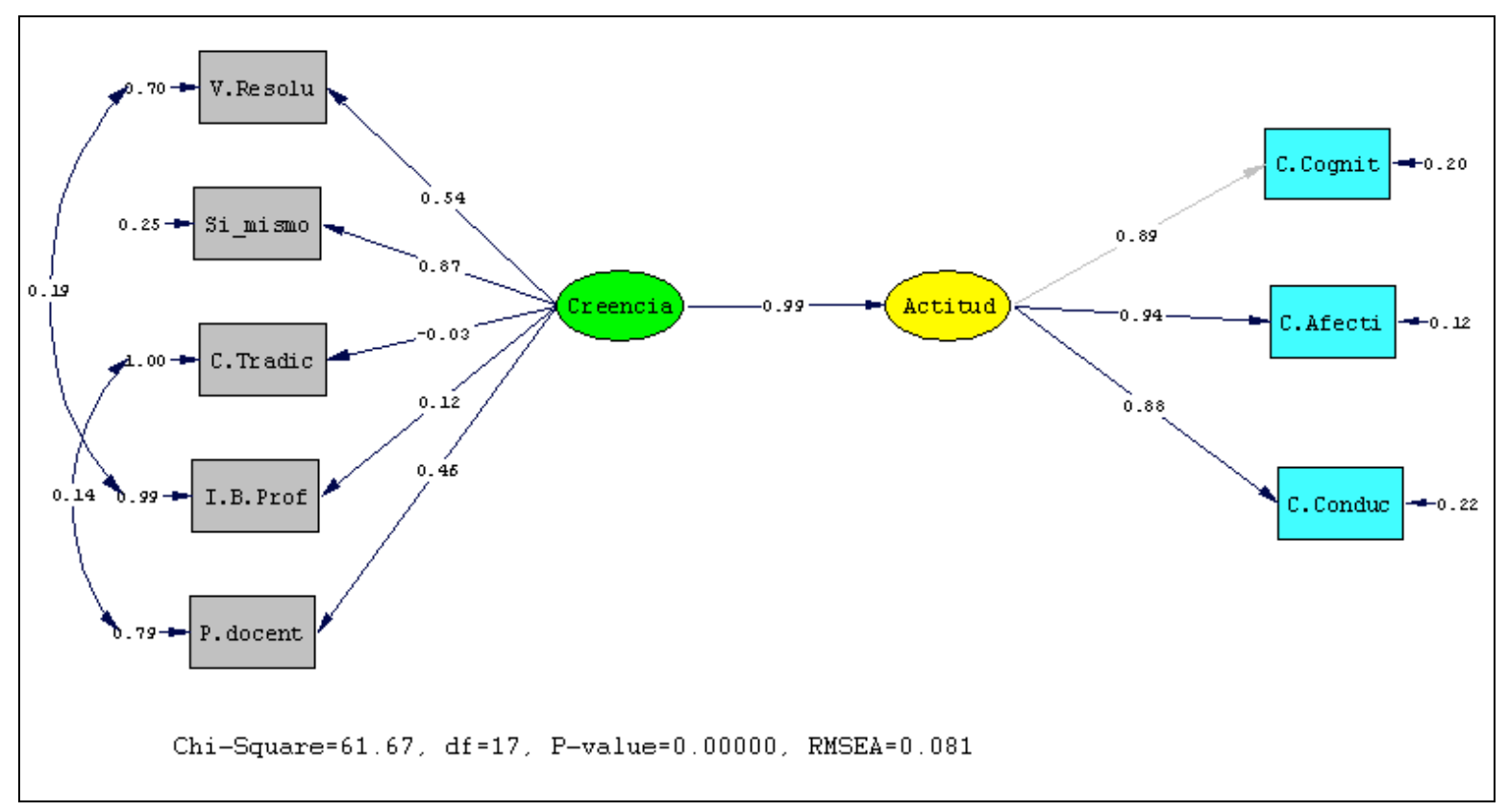

Figura 9. Coeficientes estandarizados del modelo para la dupla de visión resolución de problemas-tendencia didáctica tradicionalista.

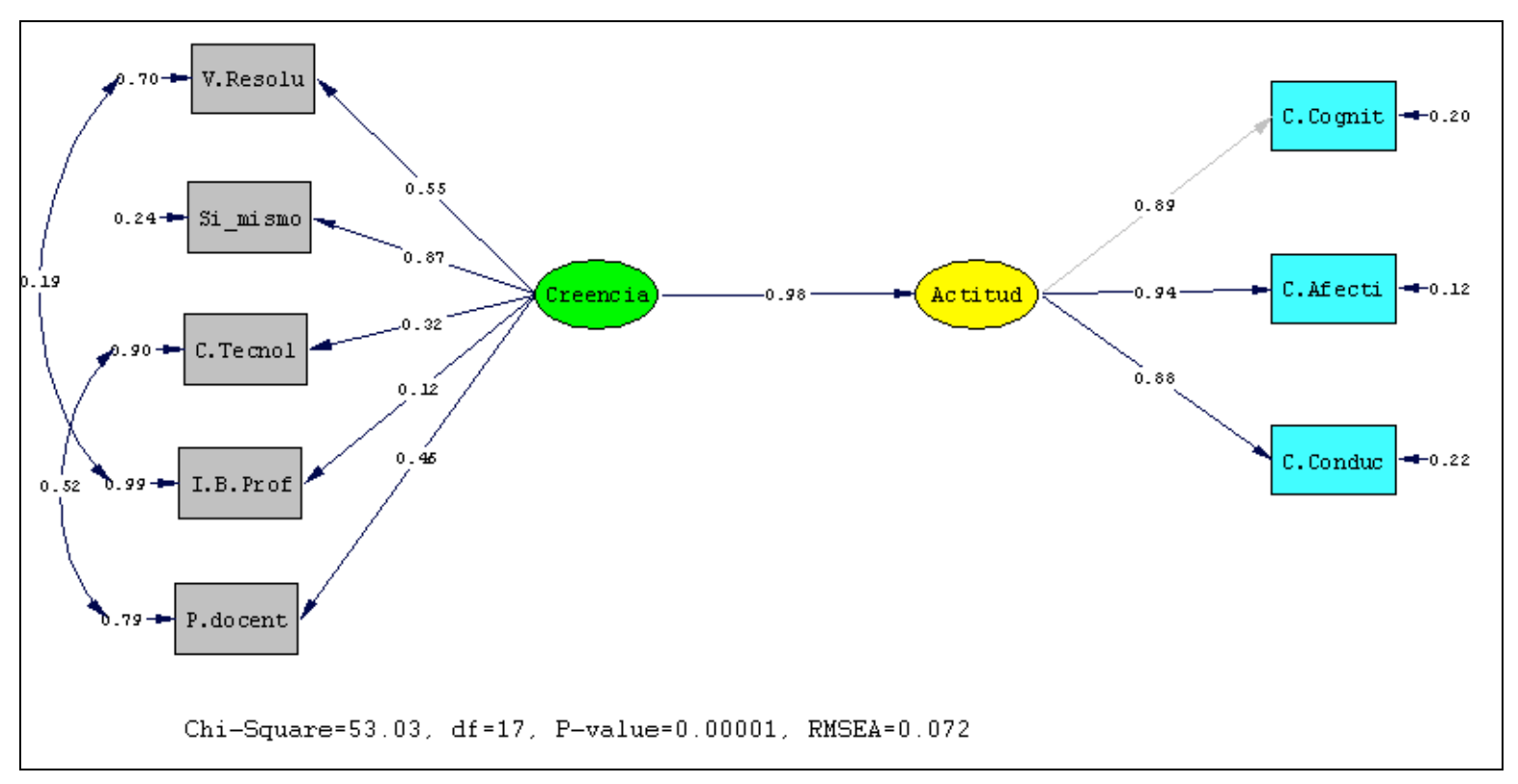

Figura 10. Coeficientes estandarizados del modelo para la dupla visión resolución de problemas- tendencia didáctica tecnológica. 


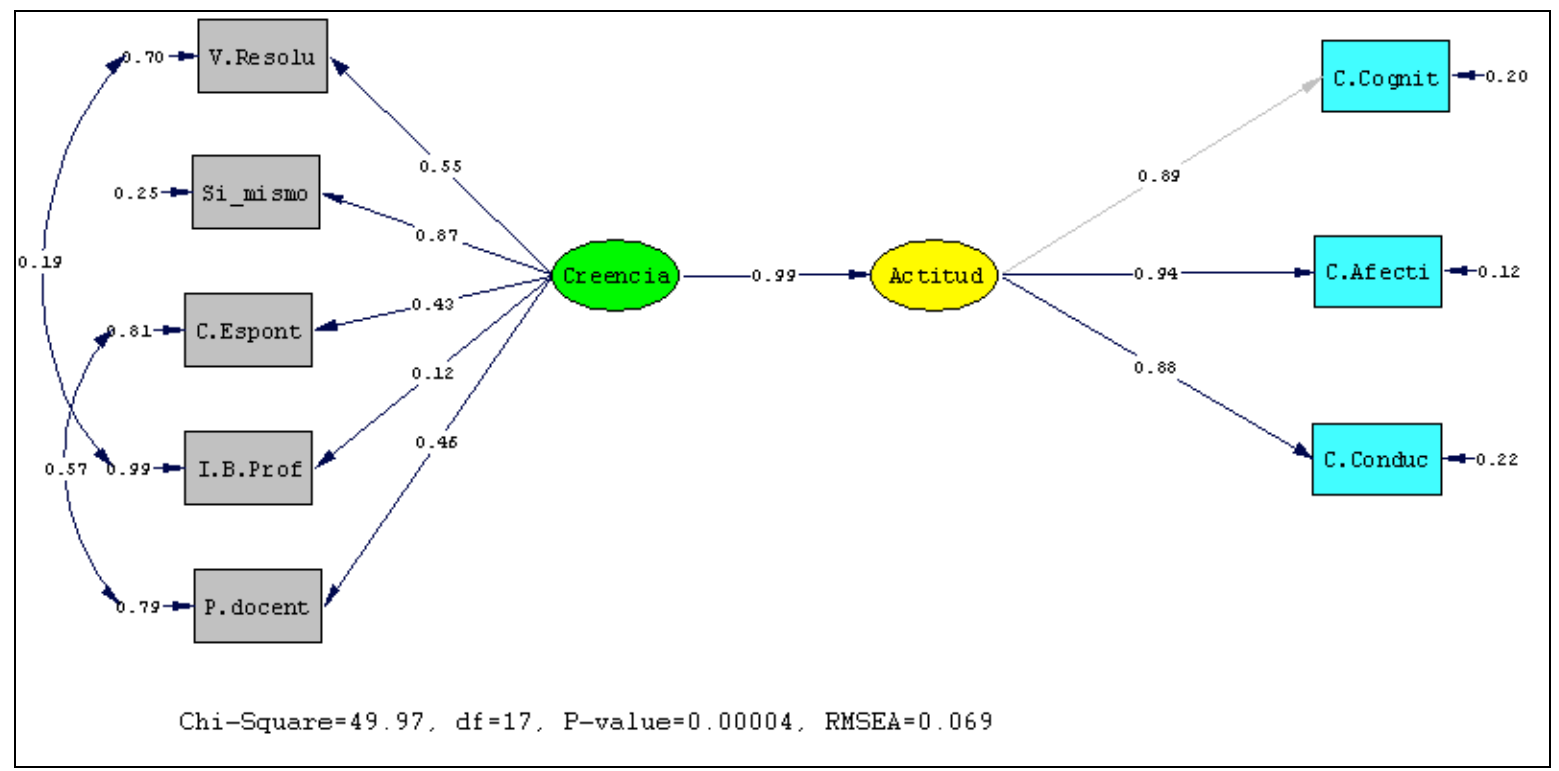

Figura 11. Coeficientes estandarizados del modelo para la dupla visión resolución de problemas- tendencia didáctica espontaneísta.

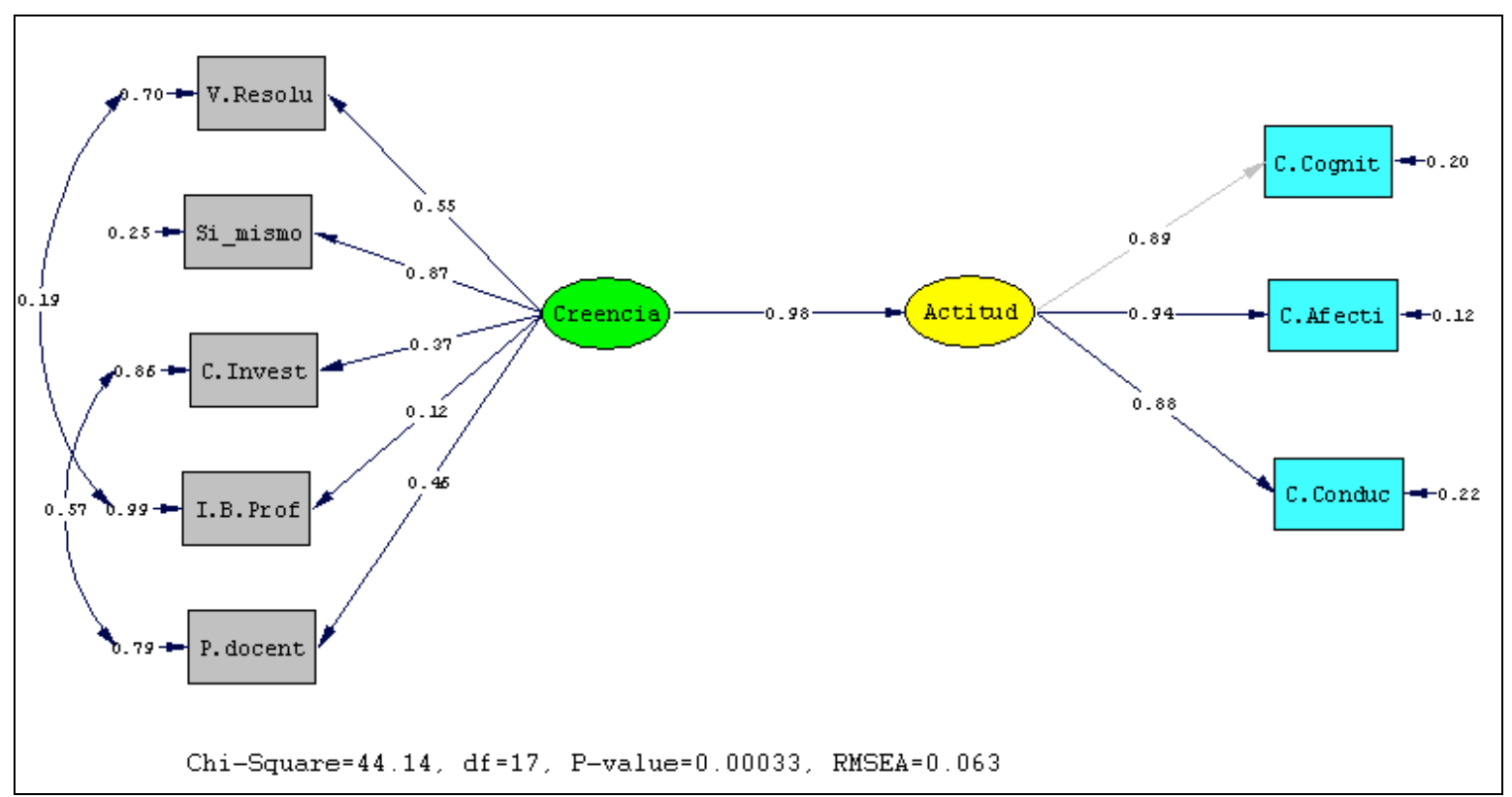

Figura 12. Coeficientes estandarizados del modelo para la dupla visión resolución de problemas-tendencia didáctica investigativa.

\subsection{Análisis de los resultados del modelo}

Es importante destacar que la interpretación de los modelos que tuvieron un buen ajuste se realiza con base en la magnitud de los coeficientes obtenidos por SatorraBlender (indicados en las líneas del modelo). En este sentido, Cea (2004) y Catena et al. (2003) apuntan que la correlación mínima propuesta, generalmente, para considerar que las variables están relacionadas es $\pm 0,30$. Es significativo mencionar que estos valores no presentan una gran diferencia con respecto a los obtenidos sin la corrección realizada, lo que representa un indicio de la robustez del modelo. 
Como es posible observar en las figuras anteriores, para el constructo actitud los valores de los tres componentes son bastantes altos, siendo el mayor el correspondiente al afectivo. Esto se presenta de igual forma en todos los modelos que obtuvieron un ajuste adecuado. Lo anterior concuerda con lo expresado por Gil et al. (2005) y Abraham et al. (2010) respecto a la constitución de dicho constructo.

Otros elementos comunes en los cuatro modelos es que el indicador relacionado con la visión que posee un estudiante respecto a sí mismo en Matemáticas es el que presenta el valor más elevado con respecto a los otros indicadores para el constructo creencia. Seguido de este, está la visión de las Matemáticas desde una concepción de resolución de problemas y la percepción del docente. Por su parte, la imagen que poseen los estudiantes respecto a lo que es un buen profesor de Matemáticas no presentó, en ningún caso, una relación considerada importante con las creencias de los estudiantes en la disciplina.

Al analizar los modelos según la tendencia didáctica del profesor, desde la perspectiva de los alumnos, fue la tendencia tradicionalista la que no mostró relación con las creencias de los estudiantes en Matemáticas, pues su valor fue -0,03. Sin embargo, para la concepción tecnológica, espontaneísta e investigativa su relación con las creencias de los estudiantes hacia las Matemáticas resultó importante, pues los valores obtenidos fueron mayores a 0,30 . Esta asociación puede ser evidencia a favor del planteamiento de causalidad.

Además de lo anterior, se encontró una asociación que puede ser evidencia de causalidad positiva entre los constructos creencia y actitud, lo que se traduce en que a mayor creencia positiva hacia las Matemáticas mejor es la actitud hacia la disciplina.

El hecho de que no exista evidencia de relación entre la tendencia tradicionalista y las creencias hacia las Matemáticas, se puede explicar, en parte, a que dicha tendencia y la visión de resolución de problemas no resultan compatibles, pues la primera se caracteriza por el uso de la exposición magistral, donde el profesor se basa en una programación prescrita con antelación, externa a él y rígida; las lecciones se centran en la adquisición de conceptos, utilizando la memoria como único recurso, donde se parte del hecho que el alumno adquiere los conocimientos a partir de la exposición que el profesor realiza, por lo que él es el único responsable de los resultados del aprendizaje (Contreras, 1998); mientras que la segunda visualiza la disciplina como un campo de creación e invención humana en continua expansión, no acabado y cuyos resultados están abiertos a revisión (Ernest, 1988), donde, según Gómez (2000), el docente es un facilitador para la construcción del conocimiento matemático y el estudiante participa activamente en este proceso.

En total se realizaron doce modelos basados en el modelo teórico propuesto; únicamente para la visión de las Matemáticas desde una concepción de resolución de problemas se obtuvo un ajuste aceptable. Bajo esta tendencia se analizaron las otras visiones de las Matemáticas, pero no fue posible obtener datos al respecto, lo cual parece razonable debido a que la visión de resolución de problemas fue con la que se identificaron un mayor número de estudiantes. Es importante señalar que lo propuesto aquí fue un modelo y no "el modelo", por lo que pueden existir otros alternativos que incorporen dichas visiones, ya que, siempre existen teorías causales alternativas que explican igualmente bien las relaciones observadas.

Una manera de comprobar si el modelo propuesto ajusta o no para dichas visiones, sería ampliar la muestra hasta tener a un número significativo de estudiantes que se 
identifican con una visión en particular y realizar el análisis propuesto. Esto es, ampliar el estudio para tres grupos distintos: los de la visión instrumentalista, aquellos que se identifican con la platónica y los que coinciden con la de resolución de problemas. Debido a que se recomiendan muestras mayores a 200 casos, este tipo de análisis no se pudo realizar en esta investigación, pues el tamaño de la muestra sería muy inferior a lo señalado.

\section{Conclusiones}

Considerando los resultados obtenidos en esta investigación y el planteamiento de las hipótesis se puede concluir que:

1. Desde la visión de las Matemáticas de resolución de problemas, existe relación entre los componentes cognitivo, afectivo y conductual y la actitud de los estudiantes hacia la disciplina. Los coeficientes de correlación fueron, respectivamente, 0,$89 ; 0,94$ y 0,88 , para todas las tendencias didácticas.

2. No se presentó evidencia de relación entre las visiones instrumentalista y platónica y las creencias de los estudiantes hacia la disciplina.

3. Existe relación entre la visión de resolución de problemas y las creencias de los estudiantes hacia la disciplina. Los coeficientes de correlación de esta visión, según las tendencias didácticas tradicionalista, tecnológica, espontaneísta e investigativa, fueron, respectivamente, 0,$54 ; 0,55 ; 0,55$ y 0,55 .

4. Desde la visión de las Matemáticas de resolución de problemas, existe relación entre la imagen de sí mismo en Matemáticas y las creencias de los estudiantes hacia la disciplina. El coeficiente de correlación fue 0,87 , para todas las tendencias didácticas.

5. Desde la visión de las Matemáticas de resolución de problemas, no se presentó evidencia de relación entre la tendencia didáctica tradicionalista del profesor y las creencias de los estudiantes hacia la disciplina. El coeficiente de correlación fue -0,03.

6. Desde la visión de las Matemáticas de resolución de problemas, existe relación entre la tendencia didáctica tecnológica, espontaneísta e investigativa del profesor de Matemáticas y las creencias de los estudiantes hacia la disciplina. Los coeficientes de correlación fueron, respectivamente, $0,32,0,43$ y 0,37 .

7. Desde la visión de las Matemáticas de resolución de problemas, no se presentó evidencia de relación entre la imagen de un buen profesor de Matemáticas, según los alumnos, y las creencias de los estudiantes hacia la disciplina. El coeficiente de correlación fue 0,12 , para todas las tendencias didácticas.

8. Desde la visión de las Matemáticas de resolución de problemas, existe relación entre la percepción del profesor de Matemáticas por parte de los estudiantes y las creencias de los alumnos hacia la disciplina. El coeficiente de correlación fue 0,46, para todas las tendencias didácticas.

9. Desde la visión de las Matemáticas de resolución de problemas, existe relación entre las creencias y actitudes de los estudiantes hacia las Matemáticas. Los coeficientes de correlación para las tendencias didácticas tradicionalista, tecnológica, espontaneísta e investigativa fueron, respectivamente, 0,$99 ; 0,98 ; 0,99$ y 0,98 .

Por otra parte, la tendencia didáctica del profesor de Matemáticas, según los estudiantes, y su relación con la percepción que estos poseen de él, resultó ser un 
factor que se relaciona con las creencias de los alumnos hacia la disciplina y, por ende, con sus actitudes. Este hecho adquiere una importancia relevante debido a que el indicador posee mayor preponderancia con las creencias hacia las Matemáticas, por parte de los estudiantes, es la imagen de sí mismos ante la disciplina.

Por lo tanto, es desde la práctica educativa de aula donde el docente puede generar estrategias para reforzar o cambiar la imagen que un estudiante posee de sí mismo. La importancia de atender el factor emocional y estar consciente de lo que el discente siente, piensa y actúa resulta un aspecto a considerar y que debe ser abordado, en la medida de lo posible, desde la labor de aula.

Obviamente existen situaciones externas a la labor de aula que afectan el aprendizaje de los estudiantes (familiares, económicas, entre otros) que requieren de un acompañamiento que el profesor no puede dar, pero el reto es hacer del aula un ambiente de aprendizaje donde estos se sientan cómodos, útiles y se estimule su aprendizaje.

Aunque existen muchas otras variables que pueden influir en las actitudes y creencias de los estudiantes hacia las Matemáticas, como las actitudes y creencias de los padres hacia la materia, la visión de los compañeros respecto al estudiante como aprendiz de la disciplina, entre otros, es importante señalar que por más complejo que se plantee un modelo de ecuaciones estructurales, es imposible incluir todos los factores que podrían tener alguna influencia en él.

Es importante resaltar que la modelación con ecuaciones estructurales trata precisamente de comprobar una o más teorías para explicar las características entre variables observadas, elemento que sí fue logrado en esta investigación pues se comprobaron las relaciones descritas entre las variables según la teoría desarrollada.

De los datos obtenidos se destaca que la mayoría de las tendencias didácticas presentaron evidencia de relación directa con las creencias hacia las Matemáticas e indirecta con las actitudes hacia la disciplina, fue la imagen del estudiante respecto a sí mismo en la materia la que mayor relación presentó $(0,87)$.

El aspecto anterior resulta de vital importancia en el proceso de enseñanza y aprendizaje de la disciplina, pues debe ser atendido de forma especial por los docentes. El profesor de Matemáticas debe conocer las necesidades que surgen del contexto del que forman parte los estudiantes, los sentimientos y pensamientos que esas necesidades generan en ellos y el impacto que las emociones originadas de esto poseen en su motivación y en su actuar (Peralta, Flores \& Otero, 2013). Lo anterior con el fin de ejecutar acciones que, desde el aula, le permitan al discente su pleno desarrollo en la materia.

Atender la parte emocional de los estudiantes debe ser una acción diaria para el equipo docente, ya que los discentes, como seres complejos, tienen emociones que están presentes en todo momento y en toda actividad, y que, por lo tanto, forman parte de su aprendizaje; sin embargo, en la clase de Matemáticas sus manifestaciones pocas veces son atendidas de manera adecuada (Peralta et al., 2013). Si las actitudes y creencias negativas o los estados de ansiedad hacia la disciplina han bloqueado la capacidad de razonamiento, es clave la toma de consciencia y la autorregulación por parte del alumno; en este sentido, la metodología del profesor es uno de los medios para lograr un cambio (Gómez-Chacón, 2009).

Lastimosamente la realidad muestra que para algunos profesores esta tarea resulta compleja, pues se concentran demasiado en cumplir el plan de estudios y el desarrollo 
de los contenidos programáticos, y apuntan los fracasos de los estudiantes a su apatía hacia la materia, sin tomar en cuenta que la angustia o pánico que pueden estar experimentando en la clase o en el examen están influyendo negativamente en ellos (Peralta et al., 2013). Por lo tanto, según los autores, no se están generando espacios para reflexionar sobre el origen de emociones como el miedo, la vergüenza, el coraje, la frustración, entre otras, y la relación de estas con el aprendizaje de los contenidos de la asignatura.

Para el docente resulta trascendental comprender la estructura del sistema de creencias de los estudiantes para ayudar a explicar algunos de sus comportamientos, así como para conocer sus capacidades, necesidades y adaptar sus estrategias de enseñanza según las exigencias del contexto (Vizcaíno \& Otero, 2012).

Desde la labor del docente en el aula es donde se pueden introducir cambios que impacten la organización, el desarrollo y evaluación de los aprendizajes, con el propósito de provocar un cambio en las actitudes y creencias de los estudiantes que impacten en la imagen de sí mismos. En este sentido, la tarea del profesor de Matemáticas debe centrarse en colaborar con el desarrollo de los alumnos y considerar a cada uno de ellos como un sujeto único, distinto y cambiante (Flores, 2004).

Desde este perspectiva, la disciplina se constituye como una área que puede propiciar "el desarrollo de actitudes relacionadas con los hábitos de trabajo, la curiosidad, el interés por investigar y resolver problemas, con la creatividad en la formulación de conjeturas, la flexibilidad para cambiar el propio punto de vista, la autonomía intelectual para enfrentarse a situaciones desconocidas y la confianza en la propia capacidad de aprender y resolver problemas" (Gómez-Chacón, 2009, p. 27). La función del docente se debe centrar, según lo anterior, en facilitar los medios para el logro de dichos comportamientos, lograr un cambio en las actitudes y creencias, propiciar una mejora en la imagen de sí mismos y contribuir con la formación integral de los discentes.

\section{Referencias}

Abraham, G., Mena, A., Rodríguez, M., Golbach, M., Rodríguez, M., \& Galindo, G. (2010). ¿La actitud hacia la matemática influye en el rendimiento académico? En P. Lestón (Ed.), Acta Latinoamericana de Matemática Educativa, 23 (pp. 75-84). México, DF: Colegio Mexicano de Matemática Educativa A. C. y Comité Latinoamericano de Matemática Educativa A. C.

Álvarez, Y. (2007). Actitudes hacia las matemáticas de los estudiantes de ingeniería de las universidades venezolanas (Tesis doctoral). Departamento de Psicología y Metodología de las ciencias del comportamiento. Facultad de Psicología. Universidad de Málaga, España.

Armenteros, B. (2009). Imagen social de las matemáticas. Las matemáticas como elemento de exclusión. Revista digital Enfoques Educativos, 30, 20-24. Recuperado el 14 de marzo de $2014 \mathrm{de}$ http://www.enfoqueseducativos.es/enfoques/enfoques_30.pdf

Blanco, L., Guerrero, E., \& Caballero, A. (2013). Cognition and Affect in Mathematics Problem Solving with Prospective Teachers. The Mathematics Enthusiast, 10(1-2), 335364.

Berenguel, E., Gil, F., Montoro, A., \&Moreno, M. (2015). Influencia de la autoconfianza y el perfil motivacional en el "flujo" en matemáticas. En C. Fernández, M. Molina \& N. Planas (eds.), Investigación en Educación Matemática XIX (pp. 173-181). Alicante: 


\section{SEIEM.}

Calabuig, F., Crespo, J., \& Mundina, J. (2012). Efecto del coste percibido, la calidad de servicio y la satisfacción sobre las intenciones futuras del espectador. Estudios de Economía Aplicada, 30(2), 619-636. Recuperado 28 de febrero de 2014 de http://redalyc.org/articulo.oa?id=30124481012

Cárdenas, C. (2008). Identificación de tipologías de actitud hacia las matemáticas en estudiantes de séptimo y octavo grados de educación primaria. Perfiles Educativos, XXX(122), 94-108.

Catena, A., Ramos, M., \& Trujillo, H. (2003). Análisis multivariado: Un manual para investigadores. Madrid, España: Editorial Biblioteca Nueva, S.L.

Casas, M. (2002). Los modelos de ecuaciones estructurales y su aplicación en el Índice Europeo de Satisfacción del Cliente. Memoria de la X Jornada de la Asociación Española de Profesores Universitarios de Matemáticas para la Economía y la Empresa. Recuperado el 14 de marzo de 2014 de http://www.uv.es/asepuma/X/C29C.pdf

Cea, M. (2004). Análisis multivariable: teoría y práctica en la investigación social. Madrid, España: Editorial Síntesis.

Chandía, E., Quiroga, F., \& Ulloa, R. (2006). Creencias de los alumnos y profesores de 1er año de enseñanza media de la intercomuna de Concepción asociadas a la asignatura de matemática. Ponencia presentada en la XIII Jornada de Investigación de Educación Matemática, Concepción, Chile. Recuperado el 19 de febrero de 2014 de http://www.sochiem.cl/jornadas2006/ponencias/05.pdf

Chaves, E., Castillo, M., \& Gamboa, R. (2008). Creencias de los estudiantes en los procesos de aprendizaje de las matemáticas. Cuadernos de Investigación y Formación en Educación Matemática, Año 3, (4), 29-44.

Contreras, L. (1998). Marco teórico sobre concepciones acerca de la enseñanza y el aprendizaje de la matemática. Capítulo 2. Tesis doctoral, Universidad de Huelva. España. $\begin{array}{lllllll}\text { Recuperado el } & 14 & \text { de } & \text { marzo }\end{array}$ http://www.uhu.es/luis.contreras/tesistexto/cap2.htm

Contreras, L. (2009). Concepciones, creencias y conocimiento: Referentes de la práctica profesional. Revista Electrónica Iberoamericana de Educación en Ciencias y Tecnología, 1(1), 11-36. Recuperado 28 de febrero de 2014 de http://www.exactas.unca.edu.ar/riecyt/VOL\%201\%20NUM\%201/Doc\%20RIECyT\%201$1 . \mathrm{pdf}$

Cubillo, L., Gamboa, N., Márquez, N., Mora, O., Montero, L., \& Fuentes, V. (2010). Actitud hacia la Matemática de las y los estudiantes de tres colegios oficiales urbanos (Memoria del Seminario de Graduación para optar por el grado de Licenciatura en la Enseñanza de la Matemática). Universidad Nacional, Costa Rica.

Demicheli, G. (2009). Calidad universitaria: actitudes y creencias de alumnos de $4^{\circ}$ de EM respecto de un subgrupo de universidades de la V región. Calidad en la Educación, 31, 93-121.

Diamantopoulo, D., \& Siguaw, J. (2000). Introducing LISREL. California,USA: SAGE Publications.

Domínguez, É., \& Jarero, M. (2010). Creencias de estudiantes de bachillerato sobre la matemática y su relación con la práctica docente. En R. Rodríguez, E. Aparicio, M. Jarero, L. Sosa, B. Ruíz, F. Rodríguez, J. Lezama \& M. Solís (Eds.), Memoria de la XIII Escuela de Invierno en Matemática Educativa (pp. 191-198). Monterrey, México: Red de Centros de Investigación en Matemática Educativa A.C.

Ernest, P. (1988). The Impact of Beliefs on the Teaching of Mathematics. En P. Ernest (Ed.), 
Mathematics Teaching: The State of the Art, 249-254. London: Falmer Press. Recuperado 28 de febrero de 2014 de http://people.exeter.ac.uk/PErnest/impact.htm

Estrada, A. (2002). Análisis de las actitudes y conocimientos estadísticos elementales en la formación del profesorado (Tesis doctoral). Departament de Didàctica de les Matemàtiques i les Ciències Experimentals. Universitat Autònoma de Barcelona. Recuperado el 14 de marzo de 2014 de http://www.tesisenxarxa.net/TDX-0502103191818/

Fernández, R. (2008). Modelos de medida y análisis factorial. En M. Verdugo, M. Crespo, M. Badía \& B. Arias (Coords.), Metodología en la investigación sobre discapacidad. Introducción al uso de las ecuaciones estructurales: VI Simposio científico SAID, 2008 (pp. 29-42). Recuperado el 19 de febrero de 2014 de http://sid.usal.es/idocs/F8/FDO20749/said 2008.pdf

Flores, P. (2004). Profesores de matemáticas reflexivos: formación y cuestiones de investigación. Actas del VIII Simposio de la Simposio de la Sociedad Española de Investigación en Educación Matemática (SEIEM), 1-16. Salamanca, España. Recuperado el 14 de marzo de 2014 de http://www.seiem.es/publicaciones/archivospublicaciones/actas/Actas08SEIEM/Flores po nencia.pdf

Gil, N., Blanco, L., \& Guerrero, E. (2005). El dominio afectivo en el aprendizaje de las Matemáticas. Una revisión de sus descriptores básicos. Revista Iberoamericana de Educación Matemática, (2), 15-32. Recuperado el 11 de abril de 2014 de http://www.fisem.org/descargas/2/Union 002 004.pdf

Gómez, I. (2000). Matemática emocional. Los efectos en el aprendizaje matemático. Madrid: Narcea Ediciones.

Gómez-Chacón, I. (2009). Actitudes matemáticas: propuestas para la transición del bachillerato a la universidad. Educación Matemática, 21(3), 5-32. Recuperado el 20 de marzo de 2014 de http://www.redalyc.org/articulo.oa? id=40516671002

Gónzalez. N., Abad, J., \& Lèvy, J. (2006). Normalidad y otros supuestos en análisis de covarianzas. En J.P. Lévy \& J. Varela (Eds.), Modelización con estructuras de covarianzas (pp. 31-57). La Coruña, España: Netbiblo.

Mora, F., \& Barrantes, H. (2008). ¿Qué es matemática? Creencias y concepciones en la enseñanza media costarricense. Cuadernos de Investigación y Formación en Educación Matemática, Año 3, (4), 71-81.

Moreira, T. (2009). Factores endógenos y exógenos asociados al rendimiento en matemática: un análisis multinivel. Educación, 33(2), 61-80. Universidad de Costa Rica, Costa Rica.

McLeod, D., \& McLeod, S. (2002). SynPAISthesis-Beliefs and Mathematics Education: Implications For Learning, Teaching, and Research. En G. Leder, E. Pehkonen \& G. Törner (Eds.), Beliefs: A hidden variable in Mathematics Education? (pp. 115-123). Londres, Inglaterra: Kluwer Academic Publishers.

Naranjo, C., \& Segura, M. (2010). Representaciones sociales de los estudiantes de la media vocacional sobre las matemáticas y la química. Memorias del VI Congreso Internacional Didácticas de las Ciencias y XI Taller Internacional sobre la Enseñanza de la Física. Simposio \# 2: La enseñanza de la Matemática en las condiciones contemporáneas: buenas prácticas y nuevas perspectivas. Recuperado 28 de febrero de 2014 de http://www.didacien.rimed.cu/Didacticas\%20de\%20las\%20Ciencias/Simposios/Simposio 2/Trabajos/Mat\%20003.pdf

Op't Eynde, P., De Corte, E., \& Verschaffel, L. (2002). Framing students' mathematics related beliefs: A quest for conceptual clarity and a comprehensive categorization. En G. Leder, E. Pehkonen y G. Törner (Eds.), Beliefs: A hidden variable in mathematics education? 
(pp. 13-38). Dordrecht, The Netherlands: Kluwer Academic Publishers.

Orgaz, M. (2008). Introducción a la metodología SEM: concepto y propósitos fundamentales. En M. Verdugo, M. Crespo, M. Badía \& B. Arias (Coords.), Metodología en la investigación sobre discapacidad. Introducción al uso de las ecuaciones estructurales: VI Simposio cientifico SAID, 2008 (pp. 15-28). Recuperado el 20 de marzo de 2014 de http://sid.usal.es/idocs/F8/FDO20749/said 2008.pdf

Padrós-Blázquez, F., Herrera-Guzmán, I., \& Gudayol-Ferré, E. (2012). Propiedades Psicométricas de la Escala de Gaudibilidad en una Población Mexicana. Revista Evaluar, 12, 1-20. Recuperado el 20 de marzo de 2014 de http://revistas.unc.edu.ar/index.php/revaluar/article/view/4693

Parra, H. (2005). Creencias matemáticas y la relación entre actores del contexto. Revista Latinoamericana de Investigación en Matemática Educativa, 8(1), 69-90. México D.F., México.

Peralta, D., Flores, D., \& Otero, M. (2013). Las Matemáticas me angustian y por eso no aprendo. Memorias del V Congreso Internacional de Educación. Universidad Autónoma de Baja California. México. Recuperado el 19 de febrero de 2014 de http://fch.mxl.uabc.mx/eventos/me5cie/cd/10113.PDF

Petriz, M., Barona, C., López, R., \& Quiroz, J. (2010). Niveles de desempeño y actitudes hacia las matemáticas en estudiantes de la licenciatura en administración en una universidad estatal mexicana. Revista Mexicana de Investigación Educativa, 15(47), 1223-1249. Consejo Mexicano de Investigación Educativa. Distrito Federal, México.

Sánchez, D. (2008). Las Creencias en la Matemática. Memoria del VI Coloquio de Experiencias Educativas en el contexto universitario, 1-12. Universidad de la Habana. La Habana, Cuba: Editorial Universitaria.

Sánchez, J., Becerra, J., García, J., \& Contreras, M. (2010). La dimensión afectiva y el rendimiento en estadística en estudiantes universitarios. En P. Lestón (Ed.), Acta Latinoamericana de Matemática Educativa, 23. México, DF: Colegio Mexicano de Matemática Educativa A. C. y Comité Latinoamericano de Matemática Educativa A. C.

Vizcaíno, A., \& Otero, I. (2012). Creencias epistemológicas y vivencias positivas en matemáticas. Revista Pensando Psicología, 8(15), 119-127. Recuperado el 19 de febrero de 2014 de http://wb.ucc.edu.co/pensandopsicologia/files/2013/07/art010-vol8-n15.pdf

Zamora, A. (2012). Validación de la prueba de diagnóstico en matemática para estudiantes de nuevo ingreso a la Universidad Nacional de Costa Rica, por medio de un modelo de Rasch y de un modelo de ecuaciones estructurales para predecir la nota en el curso de matemática general (Tesis de maestría). Sistema de estudios de posgrado. Universidad de Costa Rica. San José, Costa Rica.

\section{Referencia de los autores}

Ronny Gamboa Araya, Universidad Nacional (Costa Rica). ronny.gamboa.araya@una.cr

Tania Elena Moreira-Mora, Instituto Tecnológico de Costa Rica (Costa Rica). tmoreira@itcr.ac.cr 


\title{
An explanatory model of the beliefs and attitudes towards mathematics: An analysis based on structural equation modeling
}

\author{
Ronny Gamboa Araya, Universidad Nacional (Costa Rica). \\ Tania Elena Moreira-Mora, Instituto Tecnológico de Costa Rica (Costa Rica).
}

This article summarizes the results of an investigation whose purpose was to analyze what aspects of the beliefs and attitudes of students towards mathematics, have greater evidence of relationship with them. A quantitative design was used. Data collection was performed with 506 high school students in Costa Rica. For the analysis of the information, a structural equation model was used.

Based on the above and the proposed structural equation model, it was found that, under a vision of mathematical problem-solving, there is evidence of a relationship between the cognitive, affective and behavioral components and the students' attitude proceeding the subject, and among the students' perception about the discipline with the image of themselves within the subject, the didactic trends of Mathematics' teachers (technological, spontaneous and investigative), the teachers' perceptions and the students' attitudes toward the discipline.

Moreover, there is no evidence of a relationship between the students' perception toward the discipline with the instrumentalist and platonic view, the traditionalist teaching trend and the image of a good mathematics teacher; according to the students, the latter two aspects analyzed from a vision of mathematical problem-solving.

Most educational trends showed evidence directly related to perception about mathematics and indirectly, to the attitudes toward the discipline, under the proposed model. However, it was the self-image of the student within the discipline, which provided more connection.

The research provides important information to be considered in the teaching and learning of Mathematics, related to the perception and attitudes of students, to identify aspects that affect their formation and that should be addressed by the teacher from the classroom teaching practice. 